\title{
The effects of measurement error and omitted variables when using transition matrices to measure intergenerational mobility
}

\author{
Donal O’Neill • Olive Sweetman • Dirk Van de gaer
}

Received: 21 January 2005 / Accepted: 7 July 2006 /

Published online: 3 November 2006

(C) Springer Science + Business Media B.V. 2006

\begin{abstract}
This paper examines the consequences of specification error when transition matrices are used to analyse patterns of intergenerational mobility. We show that classical measurement error in both the child's and parent's earnings can lead to biased results, with summary mobility measures biased by as much as $20 \%$ in some cases. Furthermore our results suggest that the extent of the bias is most severe in the tails of the distribution. Omitted conditioning variables appear to have a modest effect on transition matrices in our model.
\end{abstract}

Key words transition matrices $\cdot$ specification error $\cdot$ intergenerational mobility.

\section{Introduction}

Recent work has highlighted a number of problems that arise when estimating and interpreting models of income mobility. Many of these problems relate to mismeasured or unobserved variables. For example, measurement error can occur if reported income is not an accurate measure of actual income. This problem may be compounded in models of intergenerational mobility if researchers have access to only 1 or 2 years of individual earnings, when the relevant economic variable

D. O'Neill ( $\varangle) \cdot$ O. Sweetman

Economics Dept. and NIRSA, N.U.I. Maynooth, Maynooth, Co. Kildare, Ireland e-mail: donal.oneill@nuim.ie

D. Van de gaer

SHERPPA, Ghent University, F.E.B., Vakgroep Sociale Economie,

Hoveniersberg 24, B-9000 Gent, Belgium

D. Van de gaer

CORE, 34 Voie du Roman Pays, B-1348 Louvain la Neuve, Belgium 
is lifetime earnings. Several authors have discussed measurement error bias in the context of linear regression models of intergenerational mobility (e.g., Solon [30], Zimmerman [33], Abul Naga [1] and Minicozzi [23]). Much of this work has tended to emphasise measurement error in fathers' earnings, presumably because classical measurement error in sons' earnings does not bias the OLS estimator. However, if the error process deviates from the textbook model then measurement error in sons' earnings may also be relevant (Jenkins [20], Grawe [16] and Haider and Solon [18]). Omitted variable bias, on the other hand, occurs when unobserved characteristics that are inherited from parents, such as ability, are also correlated with earnings. The OLS estimator mistakenly attributes the variation in earnings due to inherited endowments directly to parental earnings, leading us to overestimate the causal effect of parental earnings on children's earnings.

While the simple linear regression model provides a useful summary of the conditional mean function, it is only a partial description of the joint distribution of earnings. When considering intergenerational mobility patterns throughout the distribution, researchers have traditionally moved away from regression based models and relied instead upon transition matrices (Zimmerman [33], Dearden et al. [12]). However, there has been little emphasis on the consequences of specification error when this approach is adopted. For instance in an influential paper on intergenerational mobility, Zimmerman [33] uses a number of procedures to correct for potential biases in the linear regression model. However, when presenting his transition matrices he notes that " these [transition matrices] are not adjusted for measurement error, [which] could seriously alter the grouping." He goes on to warn that "[the transition matrices] should be interpreted with caution." In this paper we use a simulated model of intergenerational mobility to examine the consequences of measurement error and omitted variables on transition matrices. Although our focus is on intergenerational mobility the same issues arise when looking at individual earnings mobility across the life-cycle or country mobility over time and our findings are also relevant to these areas. We show that the intuition derived under the linear regression model should not be transposed to analyses based on transition matrices. Classical measurement error in both initial (parent's) earnings and final (son's) earnings matters when looking at the joint distribution. These errors may distort mobility patterns in the underlying model, making it more difficult for researchers to interpret their findings. In addition we show that variation in intergenerational mobility indices across countries could be explained by differences in data reliability rather than differences in the underlying structural model. For the parameter values we choose, omitted conditioning variables appear to have a relatively small effect on transition matrices.

\section{Intergenerational mobility model}

In this section we present the model of intergenerational mobility that we use throughout the paper. A son's log earnings $\left(y_{t}^{*}\right)$ is determined by parental log earnings $\left(y_{t-1}^{*}\right)$, endowments $\left(b_{t}\right)$, which are partially inherited from parents, and market luck $\left(e_{t}^{y}\right)$. Both $y_{t}^{*}$ and $y_{t-1}^{*}$ are expressed as deviations from their means. We denote the stationary variance of earnings by $\sigma_{y^{*}}^{2}$. Following Grawe and Mulligan [17] we consider endowments as the human capital that a son receives automatically

悬 Springer 
from the parent without regard for incentives. These may include genes, reputation, culture, motivation or tastes. Formally,

$$
\begin{gathered}
y_{t}^{*}=\beta y_{t-1}^{*}+\gamma b_{t}+e_{t}^{y}, \\
b_{t}=\rho b_{t-1}+e_{t}^{b},
\end{gathered}
$$

where $e_{t}^{y}$ and $e_{t}^{b}$ are iid random variables with normal distributions, mean zero and variance $\sigma_{e^{y}}^{2}$ and $\sigma_{e^{b}}^{2}$ respectively. The relationship between a son's endowment and his father's endowment is given by Eq. 2 ; $\rho$ represents the degree of inheritability of endowments. Assuming particular distributions for the stochastic components is simply a convenience which allows us to obtain analytic solutions for this model. More general distributional assumptions could be incorporated into our model using Monte Carlo methods. Nevertheless, we believe that the model we present is rich enough to highlight the pitfalls arising from specification error in transition models.

This model can be viewed as a simple mechanical model of intergenerational transfers; however it is also a special case of the model introduced by Becker and Tomes [3]. In their model parents spend resources on their own consumption, financial transfers to the child and investments in the human capital of the child. Parents are altruistic and care about their own consumption and their children's consumption. In the absence of credit constraints all children receive the efficient level of human capital, which depends on $b_{t}$. In this case the intergenerational dynamics of earnings depends only on the intergenerational dynamics of endowments (given by $\rho$ ) and $\beta$ is equal to 0 . If credit constraints exist some parents are unable to fund the efficient level of human capital. Holding $b_{t}$ constant the constraint becomes less binding as income rises, so that parents with higher incomes invest more in their children and $\beta$ is greater than 0 . In general the magnitude of $\beta$ varies across borrowing constrained families, however it is constant within this group when preferences are Cobb-Douglas. ${ }^{1}$ Therefore the model outlined in Eqs. 1 and 2 describes the intergenerational earnings dynamics for a population in which every household is credit constrained and preferences are Cobb-Douglas. While this model is somewhat restrictive, it is used in many studies of intergenerational mobility and provides a useful starting point from which to evaluate the consequences of specification error.

Although $\beta$ determines the conditional mean function of the data generating process it only provides a partial description of mobility patterns. As a result researchers have supplemented estimates of $\beta$ with transition matrices that provide a discrete summary of the distribution of sons' earnings conditional on fathers' earnings (Atkinson et al. [2], Zimmerman [33]). In contrast to the linear regression model, relatively little is known about the consequences of specification errors for estimated transition matrices. As far as we are aware there are no analytical results concerning the effects of measurement error or omitted variables for transition probabilities. There are some papers that try to account for measurement error when

${ }^{1}$ For a detailed analysis of the role of heterogeneity in intergenerational mobility models see Han and Mulligan [19]. 
measuring transitions in the labour market; for example Magnac and Visser [22] and Poterba and Summers $[27,28]$ use auxiliary information on error rates to estimate transitions between labour market states and Breen and Moisio [8] use latent class Markov models to correct for measurement error in estimating poverty transitions. The former require auxiliary data from which the truth can be ascertained, the latter requires observations on multiple transitions to identify the measurement error; these data are unlikely to be available in studies of intergenerational mobility. Other papers have used average income to control for measurement error when estimating intergenerational transition matrices (e.g., Corak and Heisz [11] and Blanden [4]). The typical time span used in this work is 2 to 5 years. However, Mazumder [24] notes that one may need to average data over a longer period to achieve reliable estimates.

In this paper we use the model given in Eqs. 1 and 2 to examine the consequences of specification error for transition matrices. We look at the impact of measurement error in both parents' and children's earnings, as well as the consequences of omitted variables. We show that knowledge of the effect of specification error on $\beta$ is not sufficient to determine the effect on transition probabilities. The paper involves no new estimation; rather we calibrate the above model using a range of estimates available from recent studies of intergenerational mobility. We use this calibrated model to examine the effects of specification error. ${ }^{2}$ The next sections outline the key features of the model for the types of specification error considered.

\subsection{Measurement error in sons' earnings}

When considering measurement error we focus on the following model :

$$
y_{t}^{*}=\lambda y_{t-1}^{*}+e_{t}^{y} .
$$

This specification is in keeping with most empirical studies that do not control for endowments, $b$, when measuring intergenerational mobility. In this case $\lambda$ is some combination of $\beta$ and $\gamma .^{3}$

Due to measurement error in the son's earnings we cannot observe $y_{t}^{*}$. Instead we observe $y_{t}$ which is defined as:

$$
y_{t}=y_{t}^{*}+u \text {. }
$$

We assume that $u$ is normally distributed with mean 0 and variance $\sigma_{u}^{2}$. We report results with and without correlation between $u$ and $y_{t}^{*}$, which we denote by $\rho_{y_{t}^{*} u}$. For the former case we choose $\rho_{y_{t}^{*} u}=-0.17 .{ }^{4}$ O'Neill et al. [25] derive

\footnotetext{
${ }^{2}$ This approach is similar to that adopted by Kim and Solon [21] when examining the implications of mean-reverting measurement error in longitudinal studies of wages and employment.

${ }^{3}$ In the event that $\gamma=0$ in Eq. 1 then $\lambda \equiv \beta$.

${ }^{4}$ This value is consistent with the findings of Bound et al. [5] who examine the extent to which current reported earnings differs from current actual earnings. In a life-cycle model, such as the one presented by Haider and Solon [18], a correlation of -0.17 occurs when sons are approximately 35 years of age.

空 Springer
} 
theoretical results for correlated measurement error in distributional analysis by considering transformations of the joint density $f_{y_{t}^{*} u}\left(y_{t}, u\right)$. We follow a similar approach when introducing correlated measurement error into our model, in that we consider transformations of the joint density conditional on parental earnings, $f_{y_{t}^{*} u y_{t-1}^{*}}\left(y_{t}, u \mid y_{t-1}^{0}\right)$. This is equivalent to specifying the correlation between $y_{t}^{*}$ and $u$ by considering only correlation between $e_{t}^{y}$ and $u$. We discuss the implications of this in more detail in Section 3.1.

A key parameter in measurement error models is the "reliability ratio", defined as $s=\frac{\operatorname{Cov}\left(y_{t}, y_{t}^{*}\right)}{\operatorname{Var}\left(y_{t}\right)}$. In the textbook model with classical measurement error this simplifies to the familiar signal to noise ratio, $\frac{\sigma_{y^{*}}^{2}}{\sigma_{y^{*}}^{2}+\sigma_{u}^{2}}$. We experiment with two values of the reliability ratio $(s): 0.5$ and 0.75 . These are within the range of estimates presented in recent studies of intergenerational mobility (Zimmerman [33] and Mazumder [24]). In our simulations we choose the variance of $y_{t}^{*}\left(\sigma_{y^{*}}^{2}\right)$ and the variance of $u\left(\sigma_{u}^{2}\right)$ to be consistent with our chosen reliability ratio and our estimate of the variance of observed earnings, $\sigma_{y}^{2}$. Throughout the paper we set $\sigma_{y}^{2}=0.3$ (see Zimmerman [33]). To examine the robustness of our results we use two different values of $\lambda: 0.5$ and $0.3^{5}$

Under our assumptions we can determine both the true density, $f_{y_{t}^{*} y_{t-1}^{*}}\left(y_{t} \mid y_{t-1}^{0}\right)$, and the misspecified density, $f_{y_{t} y_{t-1}^{*}}\left(y_{t} \mid y_{t-1}^{0}\right)$, as follows:

\section{Proposition 1}

(a) $y_{t}^{*} \mid y_{t-1}^{*}=y_{t-1}^{0} \sim N\left(\lambda y_{t-1}^{0},\left(1-\lambda^{2}\right) \sigma_{y^{*}}^{2}\right)$.

(b) $y_{t} \mid y_{t-1}^{*}=y_{t-1}^{0} \sim N\left(\lambda y_{t-1}^{0},\left(\left(1-\lambda^{2}\right) \sigma_{y^{*}}^{2}+\sigma_{u}^{2}+2 \rho_{y^{*} u} \sigma_{y^{*}} \sigma_{u}\right)\right)$.

Proof See Appendix 1.

Although the conditional means of both distributions are the same, the conditional variances differ. If $e_{t}^{y}$ and $u$ are uncorrelated, the variance of the misspecified distribution is larger than the variance of the true distribution. Negative correlation between $e_{t}^{y}$ and $u$ can partially offset this effect. This has implications for estimating mobility which we discuss later.

\subsection{Measurement error in fathers' earnings}

When considering the consequences of measurement error in the father's earnings we first assume that the true value of the son's earnings $y_{t}^{*}$, is observed. We specify the observed value of the father's earnings as:

$$
y_{t-1}=y_{t-1}^{*}+v \text {. }
$$

We assume that $v$ is normally distributed with mean zero and variance $\sigma_{v}^{2}$. We allow for the possibility that $v$ and $y_{t-1}^{*}$ are correlated, with correlation denoted

\footnotetext{
${ }^{5}$ This is consistent with the range of intergenerational elasticities reported in the literature, the majority of which are obtained by regressing the child's earnings on the parent's earnings alone.
} 
by $\rho_{y_{t-1}^{*}}$, but assume that $v$ and $y_{t}^{*}$ are uncorrelated. Under these assumptions the misspecified density, $f_{y_{t}^{*} y_{t-1}}\left(y_{t} \mid y_{t-1}^{0}\right)$ can de derived from the following Proposition:

\section{Proposition 2}

$$
y_{t}^{*} \mid y_{t-1}=y_{t-1}^{0} \sim N\left(\lambda y_{t-1}^{0} \frac{\sigma_{y^{*}}^{2}}{\sigma_{y_{t-1}}^{2}}, \sigma_{y^{*}}^{2}\left(1-\lambda^{2} \frac{\sigma_{y^{*}}^{2}}{\sigma_{y_{t-1}}^{2}}\right)\right) \text {. }
$$

Proof See Appendix 1.

In this case both the mean and the variance are biased. The direction of the biases depend on whether $\frac{\sigma_{y^{*}}^{2}}{\sigma_{y_{t-1}}^{2}} \lesseqgtr 1$. Since $\sigma_{y_{t-1}}^{2}=\sigma_{y_{t-1}^{*}}^{2}+\sigma_{v}^{2}+2 \rho_{y^{*} v} \sigma_{y^{*}} \sigma_{v}, \frac{\sigma_{y^{*}}^{2}}{\sigma_{y_{t-1}}^{2}}$ is less than 1 whenever $\rho_{y_{t-1}^{*} v}$ is greater than 0 . In this case the conditional mean is biased downwards and the conditional variance is biased upwards. However if $\rho_{y^{*}} v$ is sufficiently negative, then $\frac{\sigma_{y^{*}}^{2}}{\sigma_{y_{t-1}}^{2}}$ can exceed 1 , in which case measurement error in fathers' earnings would lead to an amplification bias in the conditional mean ${ }^{6}$ and an attenuation bias in the conditional variance.

\subsection{Simultaneous measurement error in fathers' and sons' earnings}

We also consider simultaneous measurement error in both fathers' and sons' earnings. In this case the model is specified as :

$$
\begin{aligned}
y_{t}^{*} & =\lambda y_{t-1}^{*}+e_{t}^{y}, \\
y_{t} & =y_{t}^{*}+u, \\
y_{t-1} & =y_{t-1}^{*}+v .
\end{aligned}
$$

In this section we assume that both $u$ and $v$ are normally distributed with mean 0 and variance $\sigma^{2}$. As before we conduct the analysis with two values of $s, 0.5$ and 0.75 . $^{7}$ To the best of our knowledge there have been no studies that have quantified the correlation between the errors in sons' and fathers' earnings. However results presented in Grawe [16] and Haider and Solon [18] would lead one to suspect positive correlation in intergenerational data. This is because fathers who are older than average in these samples tend to have sons who are older than average. We simulate the results for three different values of $\rho_{u v} ; \rho_{u v}=0, \rho_{u v}=0.4, \rho_{u v}=0.8$.

\footnotetext{
${ }^{6}$ For a related discussion see Haider and Solon [18]. However they only focus on bias in the conditional mean function.

${ }^{7}$ In this analysis we choose the same reliability ratio for fathers' and sons' earnings. The analysis can be easily extended to allow these to differ.
} 
When considering simultaneous measurement error we assume that $u$ and $v$ are uncorrelated with $y_{t}^{*}$ and $y_{t-1}^{*}$. Under these assumptions it is easy to show that:

\section{Proposition 3}

$y_{t} \mid y_{t-1}=y_{t-1}^{0} \sim N\left(\left[\lambda \frac{\sigma_{y^{*}}^{2}}{\sigma_{y^{*}}^{2}+\sigma^{2}}+\frac{\sigma_{u v}}{\sigma_{y^{*}}^{2}+\sigma^{2}}\right] y_{t-1}^{0},\left[\sigma_{y^{*}}^{2}+\sigma^{2}-\frac{\left(\lambda \sigma_{y^{*}}^{2}+\sigma_{u v}\right)^{2}}{\sigma_{y^{*}}^{2}+\sigma^{2}}\right]\right)$.

Proof See Appendix 1.

In this case the traditional downward bias in the conditional mean is offset by positive correlation between $u$ and $v$; the conditional variance may be under or overestimated depending on the parameters of the model.

\subsection{Omitted endowments}

To study the influence of omitted endowments we have to consider the complete intergenerational model, given by Eqs. 1 and 2. Appendix 2 contains the solution for this model. In our simulations we report results for two sets of parameters; $\beta=$ $\gamma=0.5$ and $\beta=\gamma=0.3$. Given the absence of information on $\sigma_{b}^{2}$ we assume $\sigma_{b}^{2}=$ $\sigma_{y^{*}}^{2}$. We fix $\rho=0.5$. While there is some controversy over the size of $\rho$, this value is consistent with the estimates reported by Goldberger [14].

\section{Evaluating the consequences of misspecification for the measurement of mobility}

In order to quantify the impact of specification error we need to use the distributions given in Section 2 to construct a measure of mobility. However, there is no consensus on how best to measure mobility. Fields and Ok [13] suggest that this may be because the meaning of income mobility is not well defined, with different studies focusing on different aspects of mobility. Three types of mobility are distinguished in the literature: absolute mobility, relative mobility and positional mobility. ${ }^{8}$ Absolute mobility measures are invariant to the addition of the same positive constant to both fathers' and sons' earnings and thus value movement per se. Relative mobility focuses on measures that are invariant to the multiplication of fathers' and sons' earnings by positive constants; in linear regression models this is captured by the notion of regression to the mean. In this paper we focus on transition matrices. They are associated with a third class of mobility measures; positional or ordinal measures of mobility. Such measures are invariant to any monotonic transformation of the data. We follow the approach typically adopted in empirical work and define earnings classes based on quartiles of the marginal earnings distribution. Denote the $i^{\text {th }}$ quartile in the observed earnings distribution of sons and fathers by $Q_{i}^{S}$ and $Q_{i}^{F}$

${ }^{8}$ For a recent comparison of these approaches in the context of intergenerational mobility see Checchi and Dardanoni [9]. 
respectively, where $i=1,2,3$ and define $Q_{0}^{S}=Q_{0}^{F}=-\infty$ and $\mathrm{Q}_{4}^{S}=Q_{4}^{F}=\infty$. The $(i, j)^{\text {th }}$ element of the transition matrix is defined as:

$$
p_{i j}=\operatorname{Pr}\left(Q_{i-1}^{S} \leq z_{t}<Q_{i}^{S} \mid Q_{j-1}^{F} \leq z_{t-1}<Q_{j}^{F}\right),
$$

where $z_{t}=y_{t}$ or $y_{t}^{*}$ and $z_{t-1}=y_{t-1}$ or $y_{t-1}^{*}$.

Transition matrices can be used to construct several summary measures of positional mobility (Shorrocks [29]). We report the Immobility Ratio, the Bartholomew Index and a measure based on the second largest eigenvalue of the transition matrix. We define the Immobility Ratio as the average of the diagonal elements of the transition matrix. Formally,

$$
I M=\frac{1}{n} \sum_{i=1}^{n} p_{i i},
$$

where $n$ is the number of classes.

The Bartholomew Index weights each class transition by the number of boundaries that have been crossed. In particular:

$$
B=\frac{1}{n-1} \frac{1}{n} \sum_{i=1}^{n} \sum_{j=1}^{n} p_{i j}|i-j|,
$$

The final measure is based on the second largest eigenvalue of the transition matrix $\left(E V_{2}\right)$ and is given by:

$$
L=1-\left|E V_{2}\right| \text {. }
$$

This measure captures the speed of convergence of a Markov chain towards its equilibrium distribution. As noted by Shorrocks [29] a rigid structure is associated with a slowly changing distribution such that $E V_{2}$ will be close to 1 ; a perfectly mobile structure establishes the equilibrium distribution within a single period $\left(E V_{2}=0\right)$.

\subsection{Measurement error in sons' earnings}

When looking at measurement error in sons' earnings we first consider the case where $\lambda=0.5$. Our results are given in Tables I and II. Table I presents the transition matrices and Table II provides the aggregate mobility measures. We report the results for our two chosen values of the reliability ratio. Our value of $s=0.5$ is equal to Mazumder's [24] reported reliability ratio when a single year's earnings is used to proxy for life-time earnings; $s=0.75$ corresponds to a three-year average of earnings. ${ }^{9}$ Comparing the results for these two values allows us to evaluate the effect of time-averaging on transition matrices.

The transition matrices presented in Table I are consistent with previous empirical work. The estimated matrix with classical measurement error and $s=0.5$ is very similar to that reported by Zimmerman [33] who uses a single year's earnings to proxy life-time earnings. As noted earlier Zimmerman [33] expressed a concern that measurement error might have seriously distorted his estimated transition

\footnotetext{
${ }^{9}$ See the first column of results in Table I of Mazumder [24].

空 Springer
} 
Table I Quartile group transition matrices under alternative error structures for sons $(\lambda=0.5)$

No error
$\left[\begin{array}{llll}0.481 & 0.278 & 0.168 & 0.072 \\ 0.278 & 0.296 & 0.258 & 0.168 \\ 0.168 & 0.258 & 0.296 & 0.278 \\ 0.072 & 0.168 & 0.278 & 0.481\end{array}\right]$

Classical error

Reliability ratio $(s)=0.5$

$\left[\begin{array}{llll}0.406 & 0.275 & 0.200 & 0.118 \\ 0.275 & 0.273 & 0.251 & 0.200 \\ 0.200 & 0.251 & 0.273 & 0.275 \\ 0.118 & 0.200 & 0.275 & 0.406\end{array}\right]$

Reliability ratio $(s)=0.75$

$\left[\begin{array}{llll}0.446 & 0.278 & 0.184 & 0.093 \\ 0.278 & 0.284 & 0.254 & 0.184 \\ 0.184 & 0.254 & 0.284 & 0.278 \\ 0.093 & 0.184 & 0.278 & 0.446\end{array}\right]$

Correlated error

Reliability ratio $(s)=0.5$

$\left[\begin{array}{llll}0.423 & 0.277 & 0.193 & 0.107 \\ 0.277 & 0.278 & 0.252 & 0.193 \\ 0.193 & 0.252 & 0.278 & 0.277 \\ 0.107 & 0.193 & 0.277 & 0.423\end{array}\right]$

Reliability ratio $(s)=0.75$

$\left[\begin{array}{llll}0.459 & 0.278 & 0.178 & 0.085 \\ 0.278 & 0.288 & 0.256 & 0.178 \\ 0.178 & 0.256 & 0.288 & 0.278 \\ 0.085 & 0.178 & 0.278 & 0.459\end{array}\right]$

matrices. We can gauge the magnitude of these biases by comparing the misspecified transition matrix to the true matrix. The results show that classical measurement error in sons' earnings leads us to overestimate mobility, particularly in the tails of the distribution. In the presence of classical measurement error we estimate that approximately $40.6 \%$ of sons born to fathers in the lowest quartile remain in the lowest quartile (Zimmerman's estimate was $41 \%$ ). However, when we look at the true data generating process we see that the true proportion is $48 \%$. The bias tends to be smaller in the middle of the distribution where the classical measurement error causes the proportion of stayers to fall from $29.6 \%$ to $27.3 \%$. Therefore, not only does classical measurement error in son's earnings lead to an upward bias in mobility,

Table II Aggregate mobility measures under alternative error structures in sons' earnings $(\lambda=0.5)$

\begin{tabular}{lllllllll}
\hline & \multicolumn{3}{l}{ Reliability ratio $=0.5$} & & & \multicolumn{3}{c}{ Reliability ratio=0.75 } \\
\cline { 2 - 3 } & IM & B & L & & IM & B & L \\
\hline No error & 0.388 & 0.284 & 0.561 & & 0.388 & 0.284 & 0.561 \\
Classical error & 0.340 & 0.326 & 0.692 & & 0.365 & 0.304 & 0.622 \\
Non-classical error & 0.350 & 0.317 & 0.662 & & 0.373 & 0.297 & 0.599 \\
\hline
\end{tabular}


it also distorts mobility patterns in the underlying process. Changing the reliability ratio from 0.5 to 0.75 halves the estimated bias.

The last two transition matrices in Table I examine the effect of non-classical measurement error on mobility. Given our parameter values, the negative correlation between $y_{t}^{*}$ and $u$ helps offset the bias from classical measurement error. We can draw some parallels between this finding and the established results for measurement error in the linear regression model. It is well known (e.g., Bound et al. [6]) that the OLS estimator of the slope coefficient remains unbiased when there is classical measurement error in the dependent variable. However, introducing a correlation between the error and the true value can cause a bias, with larger correlations leading to larger biases. This is because the correlation between $y_{t}^{*}$ and $u$ in the linear regression model is generated through the explanatory variables. ${ }^{10}$ However, we can also consider generating correlation between $u$ and $y_{t}^{*}$ through the stochastic term. Correlation of this type does not bias the OLS estimator since the new error term is still uncorrelated with the explanatory variables. However, O'Neill et al. [25] show that correlation of this type does matter for researchers interested in the entire distribution rather than just the mean. This is reflected in the results presented in the last two matrices of Table I. These results are consistent with Pischke [26]. He uses the PSID validation data to argue that a model which generates measurement error via transitory earnings fluctuations fits the data well. In this case correlation attenuates the role of white noise measurement error in models of earnings dynamics.

Table II summarises these transition matrices using the aggregate measures of mobility presented earlier. As expected, the results show that classical measurement error in the son's earnings leads us to overestimate mobility; the Immobility Ratio falls and the Bartholomew Index and eigenvalue measure rise. ${ }^{11}$ We can use these results to understand the extent to which measurement error may distort our ability to understand the world around us. For instance the Immobility Ratio falls by approximately five percentage points with classical measurement error. Blanden [4] reports transition matrices for the UK, the US, Germany and Canada from which we can calculate the following immobility ratios; 0.34 (UK), 0.344(US), 0.374 (Germany) and 0.30 (Canada). ${ }^{12}$ Our findings show that with the exception of the comparison between Germany and Canada, all the other differences could potentially be attributed to differences in data reliability across countries. The differences in data reliability across countries need not reflect measurement error per se. In countries with a lot of intragenerational mobility, current earnings will be a less reliable proxy for lifetime earnings. Our results in turn suggest that the corresponding estimates of intergenerational mobility will be too high in these countries. As longer panel data sets become available it will be possible to test whether these differences in

\footnotetext{
${ }^{10}$ See for example the discussion in Bound et al. [5].

${ }^{11}$ The results discussed in this section use a value of $\lambda=0.5$. Results when $\lambda=0.3$ are given in the Appendix 3 (Tables A1, A2). Qualitatively the biases are the same as those discussed in the paper though the magnitude of the bias declines as $\lambda$ falls.

${ }^{12}$ These estimates are based on father's and son's earnings averaged over 2 years but are similar to other estimates (e.g., Corak and Heisz [10]).
} 
intragenerational mobility can account for reported differences in intergenerational mobility. ${ }^{13}$

\subsection{Measurement error in fathers' earnings}

In this section we assume that the error process for fathers' earnings is similar to the one used when analysing measurement error in sons' earnings. In particular the values chosen for $\sigma_{v}^{2}$ and $\rho_{y_{t-1}^{*} v}$ are the same as those used for $\sigma_{u}^{2}$ and $\rho_{y_{t}^{*} u}$. Combined with our assumption of stationary this implies the following symmetry condition ${ }^{14}$

$$
f_{y_{t} y_{t-1}^{*}}(x, z)=f_{y_{t}^{*} y_{t-1}}(z, x) \text {. }
$$

This in turn implies that the impact of measurement error in the father's earnings on transition matrices is identical to the impact of measurement error in the son's earnings. Formally ${ }^{15}$

$$
\begin{gathered}
f_{y_{t}^{*} y_{t-1}}\left(Q_{i-1}^{S *} \leq y_{t}^{*} \leq Q_{i}^{S *} \mid Q_{j-1}^{F} \leq y_{t-1} \leq Q_{j-1}^{F}\right) \\
=f_{y_{t} y_{t-1}^{*}}\left(Q_{i-1}^{S} \leq y_{t} \leq Q_{i}^{S} \mid Q_{j-1}^{F *} \leq y_{t-1}^{*} \leq Q_{j}^{F *}\right) .
\end{gathered}
$$

\subsection{Simultaneous measurement error in fathers' and sons' earnings}

The results when both fathers' and sons' earnings are measured with error are given in Tables III and IV. We consider three values for the correlation between fathers' and sons' errors $\left(\rho_{u v}=0, \rho_{u v}=0.4, \rho_{u v}=0.8\right)$. When the errors are uncorrelated mobility rises at each quartile group of the distribution. However, if the correlation is sufficiently large mobility in the observed data is too low. As in the previous sections we see that measurement error also distorts mobility patterns within the transition matrix and again the nature of these distortions depends on the error process. Consider the results when $s=0.5$. The proportion of sons remaining in the lowest quartile group of the earnings distribution falls from 0.481 to 0.357 when $\rho_{u v}=0$; the decline in the middle is much smaller (0.296 to 0.263). However, when $\rho_{u v}=0.8$, the proportion of sons remaining in the lowest quartile group of the earnings distribution rises from 0.481 in the true distribution to 0.569 in the observed distribution; the increase in the middle is smaller, 0.296 to 0.334 . These results may have important implications for researchers interested in making inferences based on mobility patterns within transition matrices. For instance Bowles and Gintis [7]

\footnotetext{
${ }^{13}$ While the specification used throughout this section is consistent with most of the empirical work in this area we have also examined the impact of measurement error using the full model given by Eqs. 1 and 2. Following Grawe and Mulligan [17] we consider a situation where credit constraints are relatively unimportant so that all of the intergenerational earnings correlation is due to endowment transmissions. In particular we choose $\beta=0.1$ and $\gamma=0.4$. As before classical measurement error increases mobility. However the magnitude of the biases are smaller than those reported in Table II. This is because of the relatively small size of $\beta$.

${ }^{14}$ This can be easily seen from the expressions for the joint distributions given in 1 .

${ }^{15}$ For proof see Appendix 4.
} 
Table III Quartile Group Transition Matrices with simultaneous measurement error in fathers' and sons' earnings $(\lambda=0.5)$

No error
$\left[\begin{array}{llll}0.481 & 0.278 & 0.168 & 0.072 \\ 0.278 & 0.296 & 0.258 & 0.168 \\ 0.168 & 0.258 & 0.296 & 0.278 \\ 0.072 & 0.168 & 0.278 & 0.481\end{array}\right]$

Simultaneous noncorrelated error $\left(\rho_{u v}=0\right)$

Reliability ratio $(s)=0.5$

$\left[\begin{array}{llll}0.357 & 0.270 & 0.218 & 0.155 \\ 0.270 & 0.263 & 0.249 & 0.218 \\ 0.218 & 0.245 & 0.263 & 0.270 \\ 0.155 & 0.218 & 0.270 & 0.357\end{array}\right]$

Reliability $\operatorname{ratio}(s)=0.75$

$\left[\begin{array}{llll}0.417 & 0.276 & 0.196 & 0.111 \\ 0.276 & 0.276 & 0.252 & 0.196 \\ 0.196 & 0.252 & 0.276 & 0.276 \\ 0.111 & 0.196 & 0.276 & 0.417\end{array}\right]$

Simultaneous correlated error $\left(\rho_{u v}=0.4\right)$

Reliability ratio $(s)=0.5$

$\left[\begin{array}{llll}0.454 & 0.278 & 0.180 & 0.087 \\ 0.278 & 0.287 & 0.255 & 0.180 \\ 0.180 & 0.255 & 0.287 & 0.278 \\ 0.087 & 0.180 & 0.278 & 0.454\end{array}\right]$
Reliability $\operatorname{ratio}(s)=0.75$

$\left[\begin{array}{llll}0.468 & 0.278 & 0.174 & 0.079 \\ 0.278 & 0.291 & 0.256 & 0.174 \\ 0.174 & 0.256 & 0.291 & 0.278 \\ 0.079 & 0.174 & 0.278 & 0.468\end{array}\right]$

Simultaneous correlated error $\left(\rho_{u v}=0.8\right)$

Reliability ratio $(s)=0.5$

$\left[\begin{array}{llll}0.569 & 0.274 & 0.125 & 0.032 \\ 0.274 & 0.334 & 0.267 & 0.125 \\ 0.125 & 0.267 & 0.334 & 0.274 \\ 0.032 & 0.125 & 0.274 & 0.569\end{array}\right]$

Reliability ratio $(s)=0.75$

$\left[\begin{array}{llll}0.523 & 0.277 & 0.148 & 0.051 \\ 0.277 & 0.312 & 0.262 & 0.148 \\ 0.148 & 0.262 & 0.312 & 0.277 \\ 0.051 & 0.148 & 0.277 & 0.523\end{array}\right]$

Table IV Aggregate mobility measures for simultaneous measurement error $(\lambda=0.5)$

\begin{tabular}{|c|c|c|c|c|c|c|}
\hline & \multicolumn{3}{|c|}{ Reliability ratio $=0.5$} & \multicolumn{3}{|c|}{ Reliability ratio $=0.75$} \\
\hline & $\mathrm{IM}$ & B & $\mathrm{L}$ & $\mathrm{IM}$ & $\mathrm{B}$ & $\mathrm{L}$ \\
\hline No error & 0.388 & 0.284 & 0.561 & 0.388 & 0.284 & 0.561 \\
\hline$\rho_{u v}=0$ & 0.31 & 0.354 & 0.784 & 0.346 & 0.320 & 0.674 \\
\hline$\rho_{u v}=0.4$ & 0.371 & 0.299 & 0.606 & 0.379 & 0.292 & 0.584 \\
\hline$\rho_{u v}=0.8$ & 0.452 & 0.235 & 0.420 & 0.418 & 0.261 & 0.492 \\
\hline
\end{tabular}


Table V Quartile Group Transition Matrices for omitted variables $(\beta=0.5, \gamma=0.5, \rho=0.5)$

Truth, conditional

Misspecified, unconditional

$\left[\begin{array}{llll}0.558 & 0.275 & 0.131 & 0.036 \\ 0.275 & 0.328 & 0.266 & 0.131 \\ 0.131 & 0.266 & 0.328 & 0.275 \\ 0.036 & 0.131 & 0.275 & 0.558\end{array}\right] \quad\left[\begin{array}{cccc}0.580 & 0.272 & 0.119 & 0.029 \\ 0.272 & 0.340 & 0.268 & 0.119 \\ 0.119 & 0.268 & 0.340 & 0.272 \\ 0.029 & 0.119 & 0.272 & 0.580\end{array}\right]$

use intergenerational transition probabilities to suggest that "distinct mechanisms may be at work at various points of the income distribution." Our results show that measurement error makes it difficult to identify whether or not such differences exist.

Table IV reports the aggregate mobility measures based on these matrices. As expected the impact of simultaneous measurement error is much larger than that discussed in Section 3.1. When both sons' and fathers' earnings are measured with error, and this error is uncorrelated, the Immobility Ratio falls by over $20 \%$. When $\rho_{u v}=0.8$ the Immobility Ratio rises by over $15 \%$. The range of Immobility Ratios generated by these error processes now incorporates almost all the ratios derived from Blanden [4]. Thus, these cross-country differences could simply reflect differences in data reliability rather than structural differences. ${ }^{16}$

\subsection{Omitted endowments}

Tables V and VI report the effects of omitted endowments when $\beta=\gamma=\rho=0.5 .^{17}$ To identify the true causal effect of a father's earnings on his son's earnings we need to control for the transmission of endowments across generations. We do this by estimating transition matrices conditional on a specific value of the endowment. ${ }^{18}$ The misspecified model is likely to underestimate mobility, since it incorrectly attributes the effect of endowments to earnings. Comparing the transition matrices conditional on endowments with the misspecified model allows us to determine the magnitude of this bias. Given our distributional assumptions and the assumption of stationarity, the true transition matrices are identical for all endowment levels. ${ }^{19}$ The results presented in Table $\mathrm{V}$ show that, as expected, the misspecified model underestimates mobility, however the magnitude of the bias is relatively small. The proportion of sons staying in the lowest quartile group rises from 0.56 to 0.58 . The aggregate summary measures are reported in Table VI. The Immobility Ratio

\footnotetext{
${ }^{16}$ For further discussion of the differences in intergenerational mobility rates across countries see Solon [31, 32].

${ }^{17}$ Appendix 3 (Tables A3, A4) presents the simulation results when $\beta=\gamma=0.3$ and $\rho=0.5$.

${ }^{18}$ This is analagous to including measures of ability in linear regression models.

${ }^{19}$ It is important to emphasise that this is a feature of using transition matrices to measure mobility. The underlying distributions are not the same.
} 
Table VI Aggregate mobility measures for omitted variables $(\beta=0.5, \gamma=0.5, \rho=0.5)$

\begin{tabular}{llll}
\hline & IM & B & L \\
\hline True, conditional model & 0.443 & 0.241 & 0.457 \\
Misspecified, unconditional model & 0.460 & 0.229 & 0.404 \\
\hline
\end{tabular}

increases slightly from 0.443 to 0.46 when we fail to account for endowments. This is a relatively small bias compared to those reported earlier for measurement error. ${ }^{20}$

\section{Conclusion}

In this paper we examine the consequences of measurement error and omitted endowments on intergenerational transition matrices. We find that classical measurement error in the child's earnings leads us to overestimate mobility. This is in contrast to OLS, where estimates of the intergenerational earnings elasticity remain unbiased in the presence of classical measurement error in children's earnings. We also show how, in contrast to the linear regression model, negative correlation between the truth and the measurement error, may reduce the bias. The magnitude of the bias arising from measurement error is substantial when compared to the bias arising from omitted variables, particularly when we allow for measurement error in both sons' and fathers' earnings. We show that, by changing the error process, it is possible to generate a range of mobility measures consistent with those reported in previous empirical work. This raises the possibility that cross-country differences in mobility measures may reflect differences in data reliability rather than differences in the underlying structural model. In addition we show that measurement error affects not only the degree of mobility but also the pattern of mobility throughout the distribution. This finding has important implications for researchers who use differences in mobility patterns within transition matrices to make statements about the underlying model.

Acknowledgements The authors would like to thank seminar participants at the ECINEQ conference in Palma de Mallorca (2005), Andrea Ichino, the editor and four anonymous referees for helpful comments on earlier drafts of this paper. The third author acknowledges financial support from the Interuniversity Attraction Poles Programme - Belgian Science Policy [Contract No. P5/21].

\section{Appendix 1: Proofs of results in Section 2}

\section{Proof of Proposition 1}

(a)

$$
\left[\begin{array}{c}
y_{t}^{*} \\
y_{t-1}^{*}
\end{array}\right] \sim N\left[\left[\begin{array}{l}
0 \\
0
\end{array}\right], \sum_{1}\right] \text { where } \sum_{1}=\left[\begin{array}{cc}
\sigma_{y^{*}}^{2} & \lambda \sigma_{y^{*}}^{2} \\
\lambda \sigma_{y^{*}}^{2} & \sigma_{y^{*}}^{2}
\end{array}\right] \text {. }
$$

\footnotetext{
${ }^{20} \mathrm{We}$ have also examined the use of a proxy variable in place of the true ability variable and found that, as expected, the use of the proxy reduces the size of the bias. However given the relatively small size of the bias to begin with, we do not report these results. Details are available from the authors upon request.
}

空 Springer 
Applying the results for conditional normal distributions we can establish that

$$
y_{t}^{*} \mid y_{t-1}^{*}=y_{t-1}^{0} \sim N\left(\lambda y_{t-1}^{0},\left(1-\lambda^{2}\right) \sigma_{y^{*}}^{2}\right) \text {. }
$$

(b) Under our assumptions

$$
\left[\begin{array}{c}
y_{t}^{*} \\
y_{t-1}^{*} \\
u
\end{array}\right] \sim N\left[\left[\begin{array}{l}
0 \\
0 \\
0
\end{array}\right], \sum_{2}\right] \text { where } \sum_{2}=\left[\begin{array}{ccc}
\sigma_{y^{*}}^{2} & \lambda \sigma_{y^{*}}^{2} & \sigma_{y_{t}^{*} u} \\
\lambda \sigma_{y^{*}}^{2} & \sigma_{y^{*}}^{2} & 0 \\
\sigma_{y_{t}^{*} u} & 0 & \sigma_{u}^{2}
\end{array}\right] .
$$

Since any linear combination of trivariate normal random variables is also normally distributed, this implies that $y_{t}\left(=y_{t}^{*}+u\right)$ and $y_{t-1}^{*}$ are normally distributed. Under our assumptions the distribution $f_{y_{t} y_{t-1}^{*}}$ is determined by:

$$
\left[\begin{array}{c}
y_{t} \\
y_{t-1}^{*}
\end{array}\right] \sim N\left[\left[\begin{array}{l}
0 \\
0
\end{array}\right], \sum_{3}\right] \text { where } \sum_{3}=\left[\begin{array}{cc}
\sigma_{y^{*}}^{2}+\sigma_{u}^{2}+2 \sigma_{y_{t}^{*} u} & \lambda \sigma_{y^{*}}^{2} \\
\lambda \sigma_{y^{*}}^{2} & \sigma_{y^{*}}^{2}
\end{array}\right] .
$$

Applying the results for conditional normal distributions we can establish that

$$
\begin{aligned}
& y_{t} \mid y_{t-1}^{*}=y_{t-1}^{0} \sim N\left(\lambda y_{t-1}^{0},\left(1-\frac{\lambda^{2}\left(\sigma_{y^{*}}^{2}\right)^{2}}{\left(\sigma_{y^{*}}^{2}+\sigma_{u}^{2}+2 \sigma_{y_{t}^{*} u}\right) \sigma_{y^{*}}^{2}}\right)\left(\sigma_{y^{*}}^{2}+\sigma_{u}^{2}+2 \sigma_{y_{t}^{*} u}\right)\right), \\
& y_{t} \mid y_{t-1}^{*}=y_{t-1}^{0} \sim N\left(\lambda y_{t-1}^{0},\left(\left(1-\lambda^{2}\right) \sigma_{y^{*}}^{2}+\sigma_{u}^{2}+2 \sigma_{y_{t}^{*} u}\right)\right) .
\end{aligned}
$$

The result in Proposition 1 (b) follows immediately.

\section{Proof of Proposition 2}

Under our assumptions

$$
\left[\begin{array}{c}
y_{t}^{*} \\
y_{t-1}^{*} \\
v
\end{array}\right] \sim N\left[\left[\begin{array}{l}
0 \\
0 \\
0
\end{array}\right], \sum_{4}\right] \text { where } \sum_{4}=\left[\begin{array}{ccc}
\sigma_{y^{*}}^{2} & \lambda \sigma_{y^{*}}^{2} & 0 \\
\lambda \sigma_{y^{*}}^{2} & \sigma_{y^{*}}^{2} & \sigma_{y_{t-1}^{*}, v} \\
0 & \sigma_{y_{t-1}^{*}, v} & \sigma_{v}^{2}
\end{array}\right] .
$$

Since any linear combination of trivariate normal random variables is also normally distributed, this implies that $y_{t}^{*}$ and $y_{t-1}\left(=y_{t-1}^{*}+v\right)$ are normally distributed. Under our assumptions the distribution $f_{y_{t}^{*} y_{t-1}}$ is given by:

$$
\left[\begin{array}{c}
y_{t}^{*} \\
y_{t-1}
\end{array}\right] \sim N\left[\left[\begin{array}{l}
0 \\
0
\end{array}\right], \sum_{5}\right] \text { where } \sum_{5}=\left[\begin{array}{cc}
\sigma_{y^{*}}^{2} & \lambda \sigma_{y^{*}}^{2} \\
\lambda \sigma_{y^{*}}^{2} & \sigma_{y^{*}}^{2}+\sigma_{v}^{2}+2 \sigma_{y_{t-1}^{*}, v}
\end{array}\right] .
$$


Applying the results for conditional normal distributions we can establish that

$$
\begin{aligned}
y_{t}^{*} \mid y_{t-1} \sim N & \left(\lambda \frac{\sigma_{y^{*}}^{2}}{\sigma_{y^{*}}^{2}+\sigma_{v}^{2}+2 \sigma_{y_{t-1}^{*},}} y_{t-1}^{0},\right. \\
& \left.\left\{\sigma_{y^{*}}^{2}\left(1-\left(\frac{\lambda \sigma_{y^{*}}^{2}}{\left(\sigma_{y^{*}}^{2}\left(\sigma_{y^{*}}^{2}+\sigma_{v}^{2}+2 \sigma_{y_{t-1}^{*}, v}\right)\right)^{5}}\right)^{2}\right)\right\}\right) .
\end{aligned}
$$

The result in Proposition 2 follows immediately.

Proof of Proposition 3

Under our assumptions

$$
\left[\begin{array}{c}
y_{t}^{*} \\
y_{t-1}^{*}
\end{array}\right] \sim N\left[\left[\begin{array}{l}
0 \\
0
\end{array}\right], \sum_{6}\right] \text { where } \sum_{6}=\left[\begin{array}{cc}
\sigma_{y^{*}}^{2} & \lambda \sigma_{y^{*}}^{2} \\
\lambda \sigma_{y^{*}}^{2} & \sigma_{y^{*}}^{2}
\end{array}\right] .
$$

Following the earlier proof we can show that

$$
\left[\begin{array}{c}
y_{t} \\
y_{t-1}
\end{array}\right] \sim N\left[\left[\begin{array}{l}
0 \\
0
\end{array}\right], \sum_{7}\right] \text { where } \sum_{7}=\left[\begin{array}{cc}
\sigma_{y^{*}}^{2}+\sigma^{2} & \lambda \sigma_{y^{*}}^{2}+\sigma_{u v} \\
\lambda \sigma_{y^{*}}^{2}+\sigma_{u v} & \sigma_{y^{*}}^{2}+\sigma^{2}
\end{array}\right] .
$$

The result of Proposition 3 follows from the properties of conditional normal distributions and our definition of $s$.

\section{Appendix 2: The equilibrium distribution of the intergenerational model}

The intergenerational model is described by Eqs. 1 and 2, repeated here for convenience:

$$
\begin{aligned}
y_{t}^{*} & =\beta y_{t-1}^{*}+\gamma b_{t}+e_{t}^{y}, \\
b_{t} & =\rho b_{t-1}+e_{t}^{b},
\end{aligned}
$$

where $e_{t}^{y}$ and $e_{t}^{b}$ are iid with mean zero and variance $\sigma_{e^{y}}^{2}$ and $\sigma_{e^{b}}^{2}$, respectively. We assume that $0<\beta<1$ and $0<\rho<1$. We can establish the following theorem:

Theorem 1 Solution to the Intergenerational Model:

$$
\left[\begin{array}{c}
y_{t}^{*} \\
y_{t-1}^{*} \\
b_{t}
\end{array}\right] \sim N\left[\left[\begin{array}{l}
0 \\
0 \\
0
\end{array}\right], \Omega\right]
$$

where $\Omega=$

$$
\left[\begin{array}{ccc}
\gamma^{2} \frac{1}{1-\rho^{2}} \frac{1}{1-\beta^{2}}\left[\frac{1+\beta \rho}{1-\beta \rho}\right] \sigma_{e^{b}}^{2}+\frac{1}{1-\beta^{2}} \sigma_{e^{y}}^{2} \gamma^{2} \frac{1}{1-\rho^{2}} \frac{1}{1-\beta^{2}}\left[\frac{\rho+\beta}{1-\beta \rho}\right] \sigma_{e^{b}}^{2}+\frac{\beta}{1-\beta^{2}} \sigma_{e^{y}}^{2} \frac{\gamma}{\left(1-\rho^{2}\right)(1-\beta \rho)} \sigma_{e^{b}}^{2} \\
\gamma^{2} \frac{1}{1-\rho^{2}} \frac{1}{1-\beta^{2}}\left[\frac{\rho+\beta}{1-\beta \rho}\right] \sigma_{e^{b}}^{2}+\frac{\beta}{1-\beta^{2}} \sigma_{e^{y}}^{2} \gamma^{2} \frac{1}{1-\rho^{2}} \frac{1}{1-\beta^{2}}\left[\frac{1+\beta \rho}{1-\beta \rho}\right] \sigma_{e^{b}}^{2}+\frac{1}{1-\beta^{2}} \sigma_{e^{y}}^{2} \gamma \rho \frac{1}{1-\rho^{2}} \frac{1}{1-\beta \rho} \sigma_{e^{b}}^{2} \\
\frac{\gamma}{\left(1-\rho^{2}\right)(1-\beta \rho)} \sigma_{e^{b}}^{2} & \gamma \rho \frac{1}{1-\rho^{2}} \frac{1}{1-\beta \rho} \sigma_{e^{b}}^{2} & \frac{1}{1-\rho^{2}} \sigma_{e^{b}}^{2}
\end{array}\right] .
$$

Proof Available upon request.

悬 Springer 


\section{Appendix 3:}

Table A1 Quartile Group Transition Matrices under alternative error structures $(\lambda=0.3)$

Classical error

Reliability ratio $(s)=0.5$

$\left[\begin{array}{llll}0.340 & 0.268 & 0.224 & 0.168 \\ 0.268 & 0.260 & 0.248 & 0.224 \\ 0.224 & 0.248 & 0.260 & 0.268 \\ 0.168 & 0.224 & 0.268 & 0.340\end{array}\right]$

Correlated error

Reliability ratio $(s)=0.5$

$\left[\begin{array}{llll}0.350 & 0.269 & 0.221 & 0.161 \\ 0.269 & 0.262 & 0.249 & 0.221 \\ 0.221 & 0.249 & 0.262 & 0.269 \\ 0.161 & 0.221 & 0.269 & 0.350\end{array}\right]$

Simultaneous noncorrelated error $\left(\rho_{u v}=0\right)$

Reliability ratio $(s)=0.5$

$\left[\begin{array}{llll}0.313 & 0.263 & 0.232 & 0.191 \\ 0.263 & 0.256 & 0.248 & 0.232 \\ 0.232 & 0.248 & 0.256 & 0.263 \\ 0.191 & 0.232 & 0.263 & 0.313\end{array}\right]$

Simultaneous correlated error $\left(\rho_{u v}=0.4\right)$

Reliability ratio $(s)=0.5$

$\left[\begin{array}{llll}0.405 & 0.275 & 0.201 & 0.120 \\ 0.275 & 0.273 & 0.251 & 0.201 \\ 0.201 & 0.251 & 0.273 & 0.275 \\ 0.120 & 0.201 & 0.275 & 0.405\end{array}\right]$

Simultaneous correlated error $\left(\rho_{u v}=0.8\right)$

Reliability ratio $(s)=0.5$

$\left[\begin{array}{llll}0.509 & 0.278 & 0.156 & 0.058 \\ 0.278 & 0.306 & 0.261 & 0.156 \\ 0.156 & 0.261 & 0.306 & 0.278 \\ 0.058 & 0.156 & 0.278 & 0.509\end{array}\right]$

Reliability ratio $(s)=0.75$

$\left[\begin{array}{llll}0.361 & 0.271 & 0.216 & 0.151 \\ 0.271 & 0.264 & 0.249 & 0.216 \\ 0.216 & 0.249 & 0.264 & 0.271 \\ 0.151 & 0.216 & 0.271 & 0.361\end{array}\right]$

Reliability $\operatorname{Ratio}(s)=0.75$

$\left[\begin{array}{llll}0.369 & 0.272 & 0.214 & 0.146 \\ 0.272 & 0.265 & 0.249 & 0.214 \\ 0.214 & 0.249 & 0.265 & 0.272 \\ 0.146 & 0.214 & 0.272 & 0.369\end{array}\right]$

Reliability ratio $(s)=0.75$

$\left[\begin{array}{llll}0.346 & 0.269 & 0.222 & 0.164 \\ 0.269 & 0.261 & 0.248 & 0.222 \\ 0.222 & 0.248 & 0.261 & 0.269 \\ 0.164 & 0.222 & 0.269 & 0.346\end{array}\right]$

Reliability ratio $(s)=0.75$

$\left[\begin{array}{llll}0.392 & 0.274 & 0.205 & 0.128 \\ 0.274 & 0.270 & 0.250 & 0.205 \\ 0.205 & 0.250 & 0.270 & 0.274 \\ 0.128 & 0.205 & 0.274 & 0.392\end{array}\right]$

Reliability ratio $(s)=0.75$

$\left[\begin{array}{llll}0.442 & 0.278 & 0.186 & 0.095 \\ 0.278 & 0.283 & 0.254 & 0.186 \\ 0.186 & 0.254 & 0.283 & 0.278 \\ 0.095 & 0.186 & 0.278 & 0.442\end{array}\right]$


Table A2 Aggregate mobility measures under alternative error structures $(\lambda=0.3)$

\begin{tabular}{|c|c|c|c|c|c|c|}
\hline & \multicolumn{3}{|c|}{ Reliability ratio $=0.5$} & \multicolumn{3}{|c|}{ Reliability ratio $=0.75$} \\
\hline & IM & $\mathrm{B}$ & $\mathrm{L}$ & $\mathrm{IM}$ & $\mathrm{B}$ & $\mathrm{L}$ \\
\hline No error & 0.324 & 0.341 & 0.740 & 0.324 & 0.342 & 0.740 \\
\hline Classical error & 0.300 & 0.364 & 0.817 & 0.312 & 0.352 & 0.776 \\
\hline Non-classical error & 0.306 & 0.359 & 0.799 & 0.317 & 0.348 & 0.762 \\
\hline Simultaneous error $\left(\rho_{u v}=0\right)$ & 0.284 & 0.380 & 0.871 & 0.303 & 0.361 & 0.806 \\
\hline $\operatorname{Simultaneous~error}\left(\rho_{u v}=0.4\right)$ & 0.339 & 0.327 & 0.696 & 0.331 & 0.334 & 0.718 \\
\hline Simultaneous error $\left(\rho_{u v}=0.8\right)$ & 0.407 & 0.269 & 0.515 & 0.362 & 0.306 & 0.629 \\
\hline
\end{tabular}

Table A3 Transition matrices with omitted variables $(\beta=0.3, \gamma=0.3, \rho=0.5)$

Truth, conditional
$\left[\begin{array}{cccc}0.390 & 0.272 & 0.205 & 0.130 \\ 0.272 & 0.266 & 0.247 & 0.205 \\ 0.205 & 0.247 & 0.266 & 0.272 \\ 0.130 & 0.205 & 0.272 & 0.390\end{array}\right] \quad$ Misspecified, unconditional

Table A4 Aggregate mobility measures with omitted variables $(\beta=0.3, \gamma=0.3, \rho=0.5)$

\begin{tabular}{llll}
\hline & IM & B & L \\
\hline True, conditional model & 0.328 & 0.333 & 0.722 \\
Misspecified, unconditional model & 0.339 & 0.323 & 0.690 \\
\hline
\end{tabular}

\section{Appendix 4: Equivalence of measurement error in fathers' and sons' earnings}

$$
\begin{aligned}
& f_{y_{i}^{*} y_{t-1}}\left(Q_{i-1}^{S *} \leq y_{t}^{*}<Q_{i}^{S *} \mid Q_{i-1}^{F} \leq y_{t-1}<Q_{i}^{F}\right), \\
& =4 \int_{Q_{i-1}^{F}}^{Q_{i}^{F}} \int_{Q_{i-1}^{S *}}^{Q_{i}^{S *}} f_{y_{i}^{*} y_{t-1}}(x, z) d x d z .
\end{aligned}
$$

Using the fact that $f_{y_{t} y_{t-1}^{*}}(x, z)=f_{y_{t}^{*} y_{t-1}}(z, x)$ and $Q_{i}^{S}=Q_{i}^{F}$ and $Q_{i}^{S *}=Q_{i}^{F *}$ we can rewrite this as

$$
\begin{aligned}
& =4 \int_{Q_{i-1}^{F *}}^{Q_{i}^{F *}} \int_{Q_{i-1}^{S}}^{Q_{i}^{S}} f_{y_{t} y_{t-1}^{*}}(z, x) d z d x, \\
& =f_{y_{t} y_{t-1}^{*}}\left(Q_{i-1}^{S} \leq y_{t}<Q_{i}^{S} \mid Q_{i-1}^{F *} \leq y_{t-1}^{*}<Q_{i}^{F *}\right) .
\end{aligned}
$$




\section{References}

1. Abul Naga, R.: Estimating the intergenerational correlation of incomes: an errors-in-variables framework. Economica 69, 69-91 (2002)

2. Atkinson, A.B., Maynard, A.K., Trinder, C.G.: Parents and Children. Heinemann, London, UK (1983)

3. Becker, G., Tomes, N.: Human capital and the rise and fall of families. J. Labor Econ. 4, S1-S39 (1986)

4. Blanden, J.: International evidence on intergenerational mobility. Working Paper, Centre for Economic Performance, London School of Economics (2005)

5. Bound, J., Brown, C., Duncan, G., Rodgers, W.: Evidence on the validity of cross-sectional and longitudinal labor market data. J. Labor Econ. 12(3), 345-368 (1994)

6. Bound, J., Brown, C., Mathiowetz, N.: Measurement error in survey data. In: Heckman, J.J., Leamer, E. (eds.) Handbook of Econometrics, vol. 5, pp. 3707-3843 (2001)

7. Bowles. S., Gintis: The inheritance of inequality. J. Econ. Perspect. 16(3), 3-30 (2002)

8. Breen, R., Moisio, P.: Poverty dynamics corrected for measurement error. Journal of Economic Inequality 2(3), 171-191 (2004)

9. Checchi, D., Dardanoni, V.: Mobility comparisons: does using different measures matter. Res. Econ. Inequal. 9, 113-145 (2002)

10. Corak, M., Heisz, A.: The intergenerational income mobility of Canadian men. Analytical Studies Branch Research Paper no. 89, Statistics Canada (1996)

11. Corak, M., Heisz, A.: The intergenerational earnings and income mobility of Canadian Men. J. Hum. Resour. 34, 504-533 (1999)

12. Dearden, L., Machin, S., Reed, H.: Intergenerational mobility in Britain. Econ. J. 107, 47-66 (1997)

13. Fields, G., Ok, E.: The measurement of income mobility: an introduction to the literature. In: Silber, J. (ed) Handbook on Income Inequality Measurement, pp. 557-596. Kluwer, Boston (2001)

14. Goldberger, A.: Heritability. Economica 46, 327-347 (1979)

15. Grawe, N.: Reconsidering the use of non-linearities in intergenerational earnings mobility as a test for credit constraints. J. Hum. Resour. 39(3), 813-827 (2004)

16. Grawe, N.: Lifecycle Bias in Estimates of Intergenerational Earnings Persistence. Labour Economics 13(5), 551-570 (2006)

17. Grawe, N., Mulligan, C.: Economic interpretations of intergenerational correlations. J. Econ. Perspect. 6(3), 45-58 (2002)

18. Haider, S., Solon, G.: Life-cycle variation in the association between current and lifetime earnings. Am. Econ. Rev. 96(4), 1308-1319 (2006)

19. Han, S., Mulligan, C.B.: Human capital, heterogeneity, and estimated degrees of intergenerational mobility. Econ. J. 111, 207-243 (2001)

20. Jenkins, S.: Snapshots versus movies: lifecycle biases and the estimation of intergenerational earnings inheritances. Eur. Econ. Rev. 31, 1149-1158 (1987)

21. Kim, B., Solon, G.: Implications of mean-reverting measurement error for longitudinal studies of wages and employment. Rev. Econ. Stat. 87, 193-196 (2005)

22. Magnac, T., Visser, M.: Transition models with measurement errors. Rev. Econ. Stat. 81(3), 466474 (1999)

23. Minicozzi, A.: Estimation of sons' intergenerational earnings mobility in the presence of censoring. J. Appl. Econ. 18(3), 291-314 (2003)

24. Mazumder, B.: Fortunate sons: new estimates of intergenerational mobility in the United States using social security earnings data. Rev. Econ. Stat. 87(2), 235-255 (2005)

25. O'Neill, D., Sweetman, O., Van de gaer, D.: The consequences of non-classical measurement error for distributional analysis. Working Paper, Department of Economics, National University of Ireland, Maynooth, Ireland (2004)

26. Pischke, J.-S.: Measurement error and earnings dynamics : some estimates from the PSID validation study. J. Bus. Econ. Stat. 13(3), 305-314 (1995)

27. Poterba, J., Summers, L.: Reporting errors and labour market dynamics. Econometrica 54, 1319$1338(1986)$

28. Poterba, J., Summers, L.: Unemployment benefits and labour market transitions: a multinomial logit model with errors in classification. Rev. Econ. Stat. 77, 207-216 (1995)

29. Shorrocks, A.: The measurement of mobility. Econometrica 46(5), 1013-1024 (1978) 
30. Solon, G.: Intergenerational income mobility in the United States. Am. Econ. Rev. 82, 393-408 (1992)

31. Solon, G.: Intergenerational mobility in the labor market. In: Ashenfelter, O., Card, D. (eds.) Handbook of Labor Economics vol.3A, pp. 1761-1800. Elsevier, Amsterdam, The Netherlands (1999)

32. Solon, G.: Cross-country differences in intergenerational earnings mobility. J. Econ. Perspect. 16(3), 59-66 (2002)

33. Zimmerman, D.J.: Regression towards mediocrity in economic stature. Am. Econ. Rev. 82, 409$429(1992)$ 